\title{
MicroRNA-125a-5p Contributes to Hepatic Stellate Cell Activation through Targeting FIH1
}

\author{
Guojun Li ${ }^{a}$ Jing Li ${ }^{b}$ Changshui Lic Honggang Qid Peihong Dong ${ }^{\mathrm{e}}$ Jianjian Zheng ${ }^{f}$ \\ Fujun Yue
}

a'Department of Hepatology, Ningbo Yinzhou Second Hospital, Ningbo, bepartment of neurosurgery, The Affiliated Huai'an Hospital of Xuzhou Medical College and The Second People's Hospital of Huai'an, Huai'an, 'Department of Pathology, Ningbo Yinzhou Second Hospital, Ningbo, 'Department of Urology, Ningbo Yinzhou Second Hospital, Ningbo, 'Department of Infectious Diseases, The First Affiliated Hospital of Wenzhou Medical University, Wenzhou, 'Wenzhou Key Laboratory of Surgery,

The First Affiliated Hospital of Wenzhou Medical University, Wenzhou, China

\section{Key Words}

MicroRNA • MiR-125a-5p • Hepatic stellate cells • Liver fibrosis • FIH1

\begin{abstract}
Background/Aims: Emerging evidence shows that microRNAs (miRNAs) play a crucial role in the regulation of activation, proliferation and apoptosis of hepatic stellate cells (HSCs). Previous studies have indicated that miR-125a-5p is correlated with hepatitis B virus replication and disease progression. However, little is known about the biological role and underlying mechanism of miR-125a-5p in liver fibrosis. Methods: We analyzed the level of miR-125a-5p in carbon tetrachloride-induced liver fibrosis and activated HSCs. We analyzed the effects of miR-125a-5p down-regulation on HSC activation and proliferation. We also analyzed the binding of miR-125a-5p to the $3^{\prime}$-untranslated region of factor inhibiting hypoxia-inducible factor 1 (FIH1) mRNA. Results: Up-regulation of miR-125a-5p was observed in the liver tissues of fibrotic mice and activated HSCs. Down-regulation of miR-125a-5p prevented the activation and proliferation of HSCs. FIH1, a negative modulator of hypoxia inducible factor 1 , was confirmed to be a target of miR-125a-5p using the luciferase reporter assay. Further studies demonstrated that miR-125a-5p prompted the activation and proliferation of HSCs, at least in part, by down-regulating FIH1. Conclusion: Our findings shed new light on miRNAs as a promising therapeutic target in liver fibrosis.
\end{abstract}

G. Li and J. Li contributed equally to this work.

Jianjian Zheng and Fujun Yu

KARGER
Key Laboratory of Surgery, The First Affiliated Hospital of Wenzhou Medical University, No.2 FuXue lane, Wenzhou, Zhejiang, (P.R. China); and Department of Infectious Diseases, The First Affiliated Hospital of Wenzhou Medical University, No.2 FuXue lane, Wenzhou, Zhejiang (P.R. China); E-Mail 120378196@qq.com, E-Mail tjyufujun@163.com 


\section{Introduction}

Liver fibrosis, characterized by the accumulation of extracellular matrix, is a frequent outcome of the pathological response to chronic liver injury. In response to chronic liver injury, hepatic stellate cells (HSCs) are activated and differentiate into myofibroblast-like cells, and express abundant alpha smooth muscle actin ( $\alpha$-SMA) and extracellular matrix components $[1,2]$. Therefore, the activation and proliferation of HSCs are central events in the progression of liver fibrosis. However, to date, the regulatory mechanisms of HSCs are only partially understood.

MicroRNAs (miRNAs) are endogenous, short non-coding RNAs of approximately 21-25 nucleotides in length that mediate gene expression by binding to the 3 '-untranslated region ( $3^{\prime}$ UTR) of mRNAs, thus leading to the degradation or translational suppression of the corresponding mRNAs $[3,4]$. Emerging evidence indicates that miRNAs are implicated in liver fibrosis [5-7]. For instance, miR-19b is down-regulated in activated HSCs and in fibrotic liver tissues. Ectopic expression of miR-19b in activated HSCs can markedly decrease the expression of transforming growth factor- $\beta$ receptor II through direct binding to the $3^{\prime} \mathrm{UTR}$, thus preventing the activation of HSCs [8]. miRNA profiling has revealed that the miR-29 family is down-regulated in human and murine liver fibrosis compared with normal liver tissues [9]. A further study demonstrated that miR-29b attenuates liver fibrosis by inhibiting HSC activation and enhancing apoptosis by targeting the PI3K/AKT signaling pathway [10]. Our previous study indicated that miR-17-5p stimulates the progression of liver fibrosis by the activating Wnt/ $\beta$-catenin signaling pathway [11]. These studies clearly show that miRNAs play a crucial role in the regulation of activation, proliferation and apoptosis of HSCs.

miR-125a-5p has been shown to be overexpressed in many cancers [12-14]. In addition, miR-125-5p is also reported to trigger proliferation, invasion, and migration in tumor cells [15-17]. In chronic hepatitis B patients, miR-125a-5p is correlated with hepatitis B virus (HBV) replication and disease progression [18]. Our previous study indicated that serum miR-125a-5p may predict disease progression in human liver diseases [19]. However, the biological role and underlying mechanism of miR-125a-5p in liver fibrosis requires further study.

In the present study, we show that miR-125a-5p is up-regulated in the liver tissues of fibrotic mice and activated HSCs. Down-regulation of miR-125a-5p inhibited the activation and proliferation of HSCs, at least in part, by targeting factor inhibiting hypoxia-inducible factor 1(FIH1).

\section{Materials and Methods}

Liver fibrosis model

Male C57BL/6 mice (20 - 22 g) received an intraperitoneal injection of carbon tetrachloride $\left(\mathrm{CCl}_{4}\right.$; $7 \mu \mathrm{l} / \mathrm{g} /$ mouse $)$ mixed with olive oil $\left(10 \% \mathrm{CCl}_{4}\right)$. To induce liver fibrosis, $\mathrm{CCl}_{4}$ was given twice a week for 8 weeks. Control mice were treated with an equal volume of olive oil at the same time intervals. The mice were euthanized $48 \mathrm{~h}$ after the last $\mathrm{CCl}_{4}$ administration. The experiments were performed in accordance with the principles summarized in the Animal Experimentation Ethics Committee Guide for the Care and Use of Laboratory Animals and approved by the Animal Ethics Committee of Wenzhou Medical University (Wenzhou, China). Some of the liver samples were fixed, embedded in paraffin, and processed for hematoxylin-eosin (HE) staining, van Gieson (VG) staining, and Sirius Red staining. Other liver samples were snap frozen in liquid nitrogen and stored at $-80^{\circ} \mathrm{C}$ for further analysis.

Isolation of primary mouse hepatocytes

Primary mouse hepatocytes were isolated as reported previously [20]. Briefly, after liver was sequentially in situ perfused with magnesium- and calcium- free Hanks' balanced salt solution supplemented with 0.5 mM EDTA (Invitrogen) and Dulbecco's modified Eagle's medium (DMEM) supplemented with collagenase (Sigma), the liver became soft and was removed in DMEM supplemented with collagenase at 


\begin{tabular}{|c|c|c|}
\hline \multirow{2}{*}{$\begin{array}{l}\text { Cellular Physiology } \\
\text { and Biochemistry }\end{array}$} & \multicolumn{2}{|c|}{ Cell Physiol Biochem 2016;38:1544-1552 } \\
\hline & $\begin{array}{l}\text { DOI: 10.1159/000443095 } \\
\text { Published online: April 14, } 2016\end{array}$ & $\begin{array}{l}\text { O } 2016 \text { The Author(s). Published by S. Karger AG, Basel } \\
\text { www.kargercom/cpb }\end{array}$ \\
\hline
\end{tabular}

$37^{\circ} \mathrm{C}$ for $15 \mathrm{~min}$. Hepatocytes were isolated by centrifugation of the filtered homogenate at $40 \mathrm{~g}$ for $5 \mathrm{~min}$. The pellet was washed two times with DMEM and seeded with the culture medium.

\section{Cell transfection}

Primary mouse HSCs were isolated as reported previously [21]. HSCs were maintained in DMEM containing 10\% fetal bovine serum (Gibco, Carlsbad, CA, USA), $100 \mathrm{U} / \mathrm{mL}$ penicillin and $100 \mu \mathrm{g} / \mathrm{mL}$ streptomycin (Gibco). Cells were cultured at $37^{\circ} \mathrm{C}$ in a humidified $5 \% \mathrm{CO}_{2}$ incubator. The miR-125a-5p inhibitor, miR-125a-5p mimics, miRNA negative control (miR-NC), siRNA against FIH1 (si-FIH1) and siRNA control (si-Ctrl) were obtained from GenePharma Corporation (Shanghai, China). Transfections were performed using Lipofectamine 2000 (Life Technologies, Carlsbad, CA, USA) according to the manufacturer's instructions.

\section{Cell proliferation assay}

Cell proliferation was analyzed using a CCK-8 assay kit (Beyotime Biotechnology, Jiangsu, China). HSCs were maintained in 96-well plates for $24 \mathrm{~h}$. These cells were then transfected with miR-125a-5p inhibitor or si-FIH1 using Lipofectamine 2000 (Life Technologies). Approximately $48 \mathrm{~h}$ later, CCK-8 solution was added to each well in the 96 -well plates, and the cells were incubated for another $2 \mathrm{~h}$ at $37^{\circ} \mathrm{C}$. Absorbance was measured at $450 \mathrm{~nm}$ on a microplate reader (Bio-Rad, Hercules, CA, USA).

\section{Luciferase reporter assay}

HEK293T cells were co-transfected with either luciferase reporter plasmid harboring wild-type FIH1 3'UTR (pmirGLO-FIH1-wt) or mutant FIH1 3'UTR (pmirGLO-FIH1-mut) together with miR-125a-5p mimics or miR-NC. The 3'UTR of FIH1 was cloned downstream of the firefly luciferase gene. If there was an interaction between miR-125a-5p and the 3'UTR of FIH1 mRNA, the activity of firefly luciferase was decreased. If there was no interaction, the activity of firefly luciferase was unchanged. Approximately $48 \mathrm{~h}$ after transfection, the cells were harvested and luciferase activity was determined by a luminometer using a Dual-Luciferase Reporter Assay System (Promega, Madison, WI, USA).

\section{Quantitative real-time PCR}

To examine mRNA expression, total RNA was isolated from HSCs using the TRIzol Kit (Life Technologies). Fifty nanograms of total RNA were then reverse-transcribed to cDNA using the PrimeScript ${ }^{\mathrm{TM}} 1$ st Strand cDNA Synthesis Reagent (TaKaRa Biotechnology, Dalian, China) following the manufacturer's protocols. To examine miRNA expression, total RNA was isolated using a miRNeasy Mini Kit (Qiagen, Valencia, CA, USA) according to the manufacturer's protocols. Gene expression was quantified by real-time PCR using the KAPA SYBR FAST qPCR Kit (KapaBiosystems, Boston, MA, USA), cDNA and gene-specific primers (Table 1). The relative abundance of mRNAs and miRNAs were normalized to GAPDH and U6 snRNA, respectively.

\section{Western blot}

The antibodies used in this experiment were as follows: collagen 1a1 (Col1A1) (Sigma-Aldrich, St Louis, MO, USA), $\alpha$-SMA, FIH1, hypoxia-inducible factor 1 alpha (HIF-1 $\alpha$ ) and $\beta$-actin (Abcam, Cambridge, MA, USA). The protein samples were separated by SDS-PAGE and transferred to a PVDF membrane (Millipore, Billerica, MA, USA). The membranes were incubated with the primary antibodies overnight at $4^{\circ} \mathrm{C}$ and then with the secondary antibodies at $37^{\circ} \mathrm{C}$ for $1 \mathrm{~h}$. After the membranes were washed three times with PBS-T, they were analyzed using an Odyssey infrared scanner (LI-COR Biosciences Inc., Lincoln, NE, USA).

Table 1.List of primer sequences

\begin{tabular}{lll}
\hline Gene & & Sequences $\left(5^{\prime}\right.$-3') \\
Mouse & Forward & TGCACCACCACTGCTTAG \\
Mouse & Reverse & GGATGCAGGGATGATGTTC \\
Col1A1 & Reverse & GCTGAAGTCATAACCGCCACTG \\
Mouse & Forward & TCCCTGGAGAAGAGCTACGAACT \\
$\alpha$-SMA & Reverse & AAGCGTTCGTTTCCAATGGT \\
Mouse & Forward & AGGCATTTGCAGCCGTTC \\
FIH1 & Reverse & CTAGGCTGAGTCAGGCAGTATC \\
Mouse & Forward & TCATCAGTTGCCACTTCC \\
HIF-1 $\alpha$ & Reverse & CACCATCACAAAGCCATCTAGG \\
Mouse & Forward & CTCGCTTCGGCAGCACA \\
U6 & Reverse & AACGCTTCACGAATTTGCGT \\
Mouse & Forward & ACACTCCAGCTGGGTCCCTGAGACCCTTTA \\
miR-125a-5p & Reverse & TGGTGTCGTGGAGTCG \\
& RT & CTCAACTGGTGTCGTGGAGTCGGCAATT \\
& & CAGTTGAGTCACAGGT \\
\hline
\end{tabular}




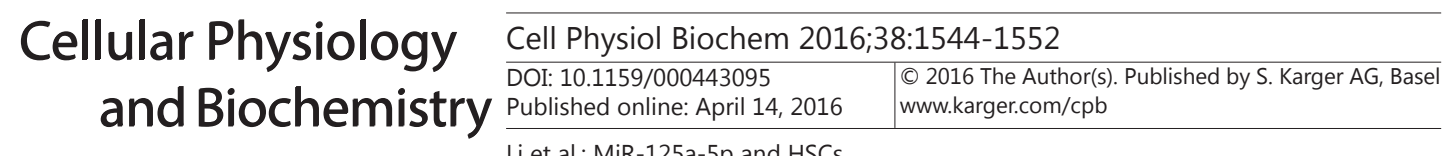

Statistical Analysis

The results are reported as the mean \pm SD. Data are compared among the groups using the Student's $t$-test or one-way analysis of variance, and $P<0.05$ was considered statistically significant. All statistical analyses were carried out using SPSS 13.0 (IBM, Armonk, NY, USA).

\section{Results}

miR-125a-5p is up-regulated in liver fibrosis and activated HSCs

miR-125a-5p has been reported to be increased in human fibrotic liver tissues [18]. To determine the level of miR-125a-5p in liver tissues from mice with $\mathrm{CCl}_{4}$-induced liver fibrosis, the severity of liver fibrosis was analyzed by HE staining, VG staining, and Sirius Red staining (Fig. 1A-C). The results indicated that 8 weeks of $\mathrm{CCl}_{4}$ administration resulted in obvious fibrous septa in liver tissues. Compared with the control, an increased level of miR125a-5p was observed in liver fibrosis (Fig. 1D), consistent with previous results in humans $[18,19]$.

Activation and proliferation of HSCs are crucial factors leading to the progression of liver fibrosis. Therefore, we examined the level of miR-125a-5p in primary HSCs and hepatocytes isolated from mice with $\mathrm{CCl}_{4}$-induced liver fibrosis. As shown in Fig. 1E, F, in parallel with the control, the level of miR-125a-5p was markedly increased in HSCs isolated from mice treated with $\mathrm{CCl}_{4}$, whereas there was a slight increase in hepatocytes isolated from mice treated with

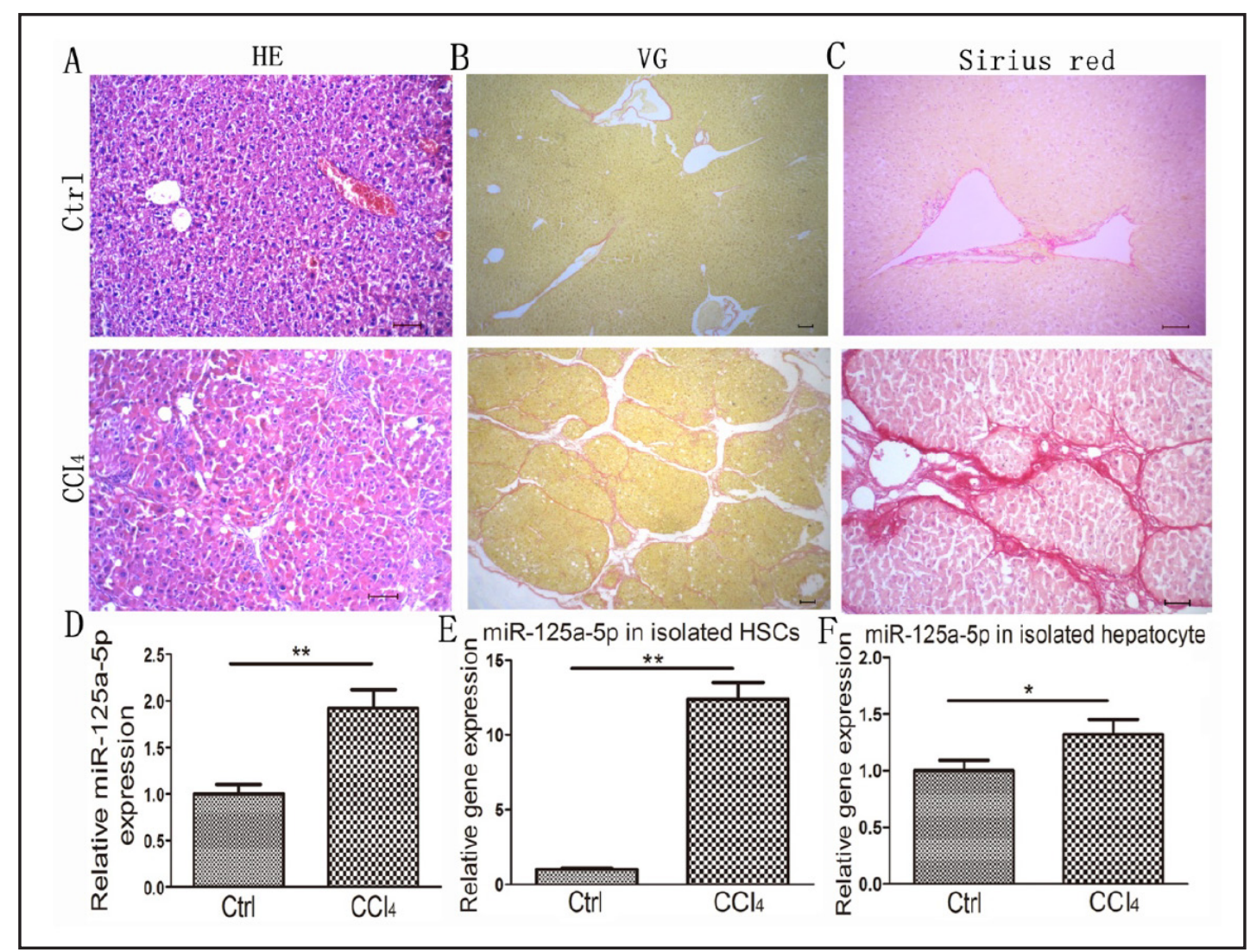

Fig. 1. Expression of miR-125a-5p is increased in liver fibrosis and activated HSCs. (A-C) HE staining, VG staining, and Sirius Red staining for the evaluation of liver fibrosis $(n=6)$. Scale bars, $100 \mu \mathrm{m}$. (D) miR-125a$5 \mathrm{p}$ expression was detected in $\mathrm{CCl}_{4}$-induced fibrotic liver tissues and normal liver tissues $(\mathrm{n}=6)$. (E) miR125a-5p expression was detected in HSCs isolated from olive oil/CCl -treated mice $(n=3)$. (F) miR-125a-5p expression was detected in hepatocytes isolated from olive oil/ $\mathrm{CCl}_{4}$-treated mice $(n=3)$. Data are presented as mean $\pm \mathrm{SD},{ }^{*} P<0.05,{ }^{* *} P<0.01$.

\section{KARGER}




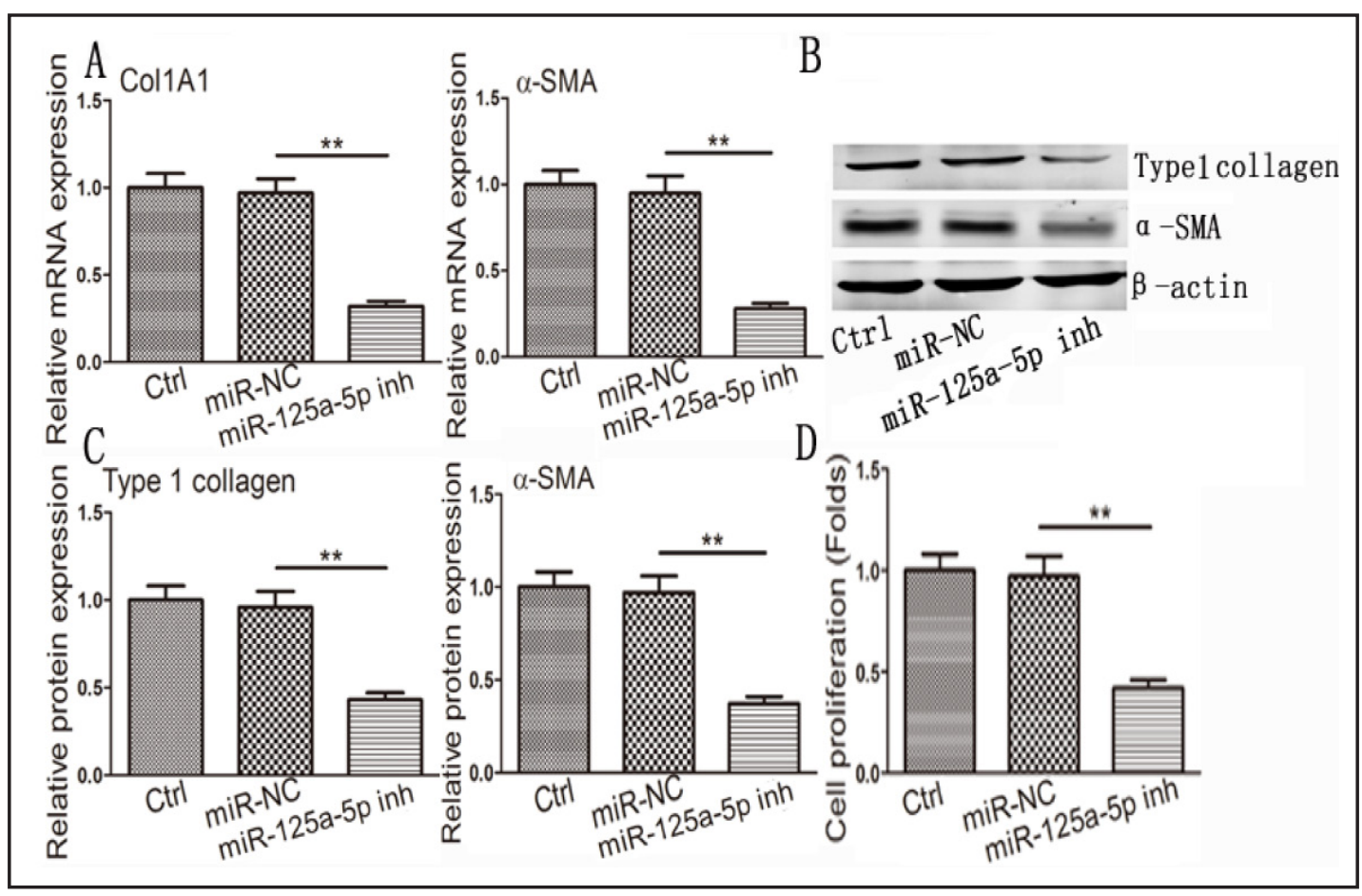

Fig. 2. Down-regulation of miR-125a-5p prevents activation and proliferation of HSCs. (A) miR-125a-5p inhibitor decreased the mRNA expression of Col1A1 and $\alpha$-SMA. (B, C) miR-125a-5p inhibitor decreased the protein expression of type 1 collagen and $\alpha$-SMA. (D) miR-125a-5p inhibitor decreased the proliferation of HSCs. Results were analyzed using the CCK-8 assay. Data represent the mean \pm SD of three independent experiments, ${ }^{* *} P<0.01$. inh, inhibitor.

$\mathrm{CCl}_{4}$.Taken together, these results indicate that miR-125a-5p is up-regulated in liver fibrosis and activated HSCs.

Effects of miR-125a-5p down-regulation on activation and proliferation of HSCS

We next determined the effects of miR-125a-5p down-regulation on the activation of HSCs. As shown in Fig. 2A, the level of Col1A1 mRNA in HSCs transfected with the miR$125 a-5 p$ inhibitor was decreased by $67 \%$ compared with the miR-NC. There was also a $71 \%$ decrease in the level of $\alpha$-SMA mRNA. Consistent with the results of mRNA levels, western blotting results indicated that the protein levels of type 1 collagen and $\alpha$-SMA were notably inhibited by the miR-125a-5p inhibitor (Fig. 2B, C). We next investigated the effects of miR$125 a-5 p$ down-regulation on the proliferation of HSCs using the CCK-8 assay. Our results demonstrated that the miR-125a-5p inhibitor triggered a 57\% reduction in HSC proliferation compared with the miR-NC (Fig. 2D). Collectively, our data suggest that down-regulation of miR-125a-5p suppresses the activation and proliferation of HSCs.

FIH1 is a target of miR-125a-5p

To further investigate the possible mechanism of miR-125a-5p in the progression of liver fibrosis, the prediction of miRNA target regions by TargetScan software (http://www. targetscan.org/) suggested that miR-125a-5p interacts with the 3'UTR of mouse FIH1 mRNA (Fig. $3 \mathrm{H}$ ). FIH1 can negatively regulate the expression of HIF-1 $\alpha$, which has been reported to be involved in liver fibrosis [22]. Our results demonstrated that the level of FIH1 mRNA was decreased in liver fibrosis and activated HSCs (Fig. 3A, B). We then determined whether miR125a-5p influenced the expression of FIH1. Similar to the miR-NC, the mRNA and protein levels of FIH1 in HSCs transfected with miR-125a-5p mimics were decreased by $69 \%$ and 


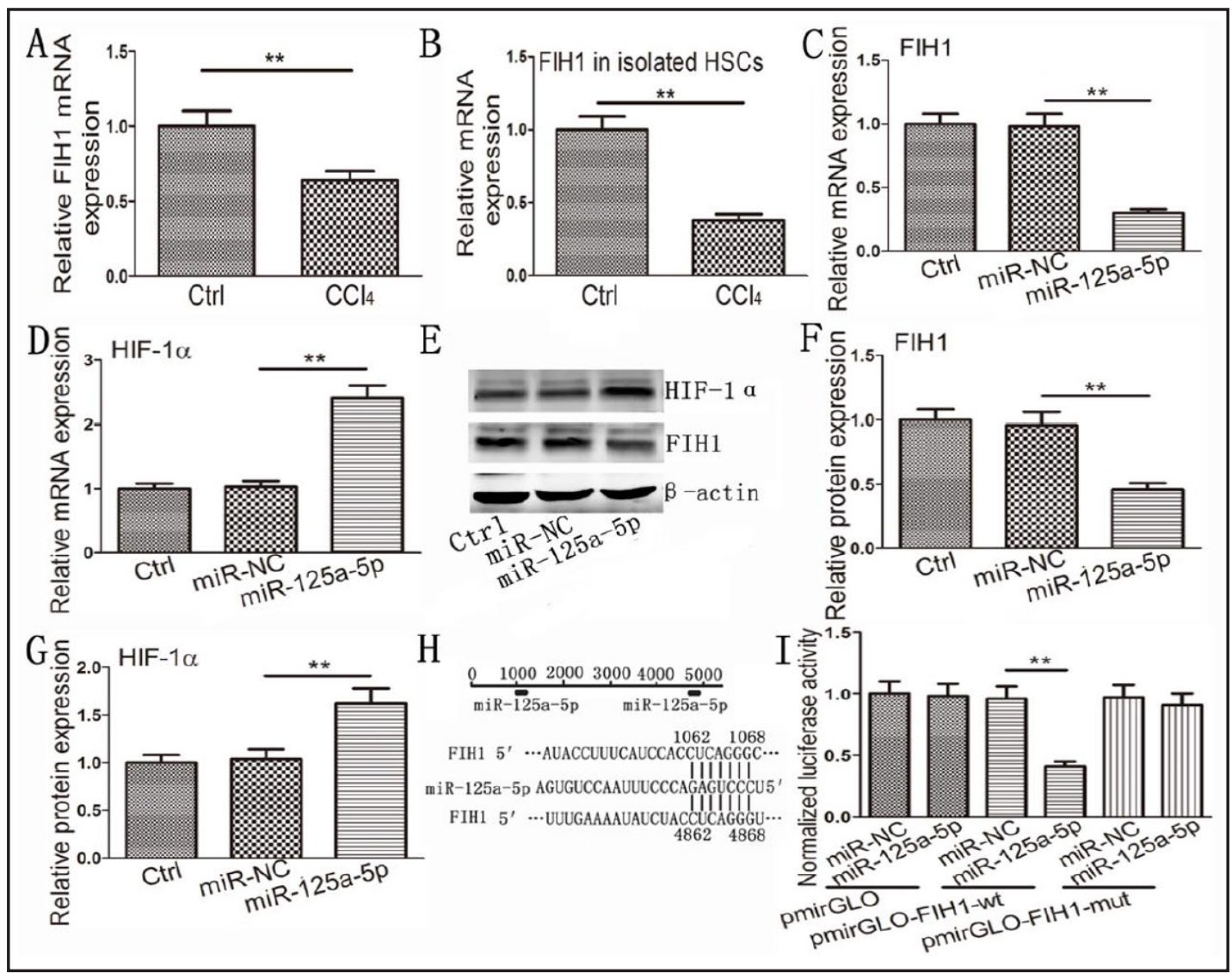

Fig. 3. Interaction of miR-125a-5p with the 3'UTR of FIH1 mRNA. (A) The mRNA expression of FIH1 was detected in $\mathrm{CCl}_{4}$-induced fibrotic liver tissues and normal liver tissues $(n=6)$. (B) The mRNA expression of FIH1 was detected in HSCs isolated from olive oil/CCl - -treated mice $(n=3)$. (C) miR-125a-5p mimics decreased the mRNA expression of FIH1 $(n=3)$. (D) miR-125a-5p mimics increased the mRNA expression of HIF-1 $\alpha(n=3)$. (E-G) miR-125a-5p mimics decreased the protein expression of FIH1 and increased the protein expression of HIF-1 $\alpha(n=3)$. (H) miR-125a-5p-binding sites in the 3'UTR of FIH1 mRNA based on TargetScan software. (I) Analysis of luciferase activity in HEK293T cells transfected with pmirGLO-FIH1-wt or pmirGLO-FIH1-mut as well as those co-transfected with miR-125a-5p mimics or miR-NC $(\mathrm{n}=3)$. Data represent the mean $\pm \mathrm{SD},{ }^{* *} P<0.01$.

$52 \%$, respectively (Fig. 3C, E and F). In contrast, the mRNA and protein levels of HIF-1 $\alpha$ were increased by 1.3 -fold and 60\%, respectively (Fig. 3D, E and G). These data indicated that the FIH1/HIF-1 $\alpha$ signaling pathway was mediated by miR-125a-5p in HSCs. To assess whether miR-125a-5p directly represses FIH1 expression via the putative miR-125a-5p binding sites, the 3'UTR target sequence of FIH1 mRNA was cloned into the pmirGLO plasmid. The construct was transfected into HEK293T cells together with miR-125a-5p mimics and miRNC. In pmirGLO-FIH1-wt, miR-125a-5p mimics led to an obvious reduction in luciferase activity compared with the miR-NC (Fig. 4I). However, in pmirGLO-FIH1-mut, miR-125a-5p mimics did not decrease luciferase activity compared with the miR-NC. In summary, our data indicate that FIH1 is a direct target of miR-125a-5p.

Down-regulation of miR-125a-5p inhibits activation and proliferation of HSCs by increasing FIH1

Hu et al. [22] reported that FIH1 is able to prevent the activation of HSCs. Thus, we examined whether FIH1 is required for the activation of HSCs induced by miR-125a-5p. As shown in Fig. 4A, B, si-FIH1 markedly decreased the mRNA and protein levels of FIH1. 


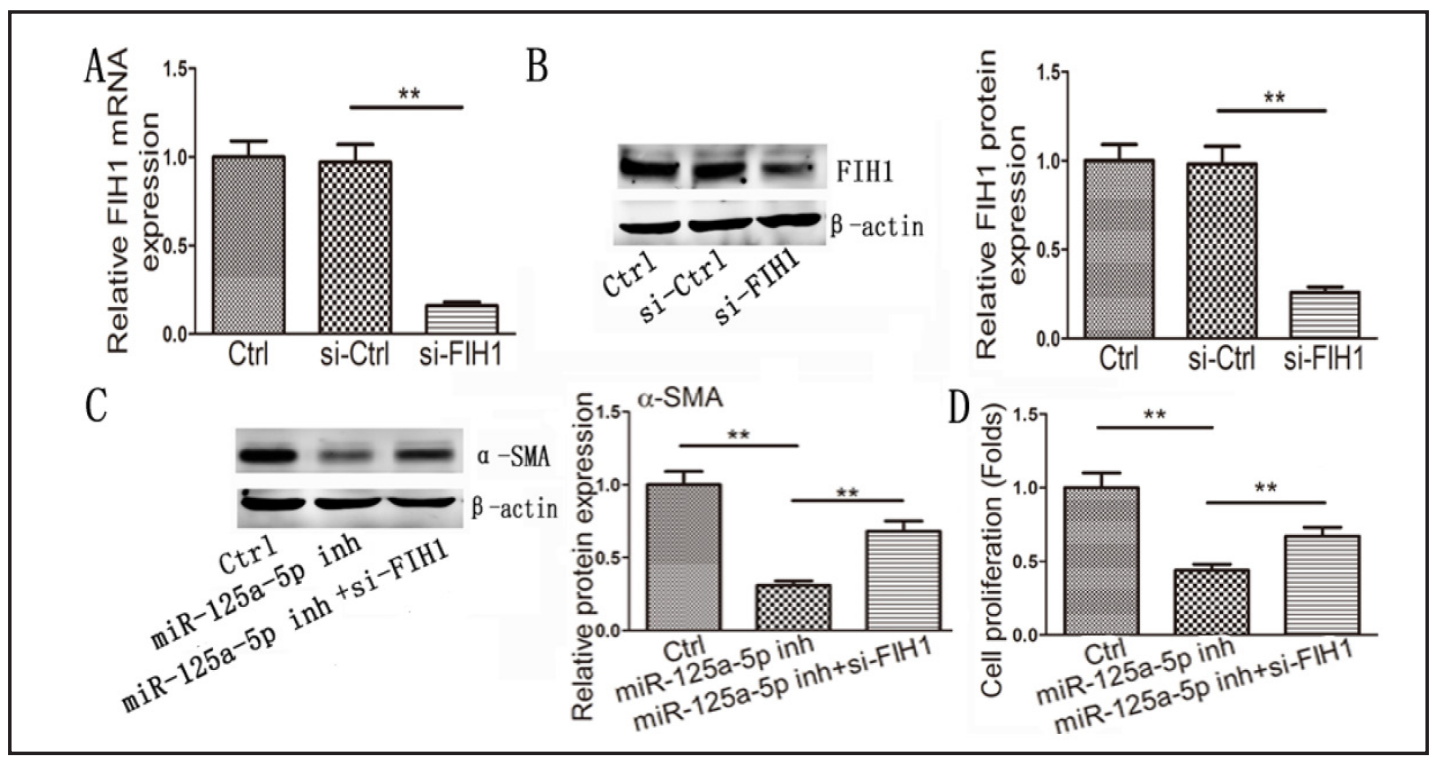

Fig. 4. miR-125a-5p inhibitor prevents activation and proliferation of HSCs by up-regulating FIH1. (A) si-FIH1 decreased the mRNA expression of FIH1. (B) si-FIH1 decreased the protein expression of FIH1. (C) Down-regulation of $\alpha$-SMA protein by miR-125a-5p inhibitor was attenuated by si-FIH1. (D) Down-regulation of HSC proliferation by miR-125a-5p inhibitor was attenuated by si-FIH1. Data represent the mean \pm SD of three independent experiments, ${ }^{* *} P<0.01$. inh, inhibitor.

In addition, the decrease in cell activation by the miR-125a-5p inhibitor was partially reversed in the presence of si-FIH1 (Fig. 4C). We next investigated whether FIH1 is required for the proliferation of HSCs induced by miR-125a-5p. Our results demonstrated that decreased cell proliferation by the miR-125a-5p inhibitor was also partially restored by the addition of si-FIH1 (Fig. 4D). In conclusion, our data indicate that miR-125a-5p promotes the activation and proliferation of HSCs, at least in part, by decreasing FIH1.

\section{Discussion}

In this study we present evidence to show that miR-125a-5p has a biological role in HSCs. Mechanistically, miR-125a-5p promotes the activation and proliferation of HSCs, at least in part, by reducing FIH1 expression. Our findings extend the field of miRNA research in liver fibrosis.

A number of studies have indicated that miR-125a-5p is involved in a variety of diseases [23-25]. miR-125a-5p expression can be induced by GATA binding protein 3 under inflammatory conditions, thus reducing the sensitivity of regulatory $\mathrm{T}$ cells by suppressing IL-6 receptor and STAT3 expression [26]. In gastric cancer, miR-125a-5p can prevent the proliferation of tumor cells and act as an independent prognostic factor [13]. In breast cancer, serum miR-125a-5p expression is a useful prognostic biomarker and provides a novel therapeutic approach by targeting HDAC4 [14]. With regard to HBV, it has been reported that miR-125a-5p can bind the viral transcript encoding the surface antigen, thus interfering with its expression and repressing viral replication [27]. Coppola et al. [18] further indicated that liver miR-125a-5p is correlated with HBV replication and disease progression in chronic hepatitis B patients. Our previous study demonstrated that serum miR-125a-5p may be a non-invasive biomarker for predicting disease progression in human liver diseases [19]. Here we report that miR-125a-5p is up-regulated in mice with liver fibrosis and activated HSCs, in line with the results obtained in humans. In addition, our results demonstrated that the addition of miR-125a-5p inhibitor hinders the activation of HSCs. Data from the proliferation assay showed that down-regulation of miR-125a-5p reduces the proliferation 
of HSCs. To the best of our knowledge, this is the first report to show that miR-125a-5p is involved in HSC activation and proliferation.

Recently, a large number of studies have demonstrated that hypoxia and HIF- $1 \alpha$ are involved in liver fibrosis. It has been reported that exposure of primary mouse HSCs to $0.5 \%$ oxygen can induce HIF-1 $\alpha$ expression, thus activating abundant genes which are pivotal for HSC function, angiogenesis, and collagen synthesis [28]. In vitro experiments showed that HIF- $1 \alpha$ knockdown can inhibit HSC activation by cooperating with mitogen activated protein kinase [29]. Data from HIF-1 $\alpha$-deficient mice demonstrate that the levels of type 1 collagen and $\alpha$-SMA were substantially decreased compared with control mice after bile duct ligation [30]. Deng et al. [31] reported that HIF- $1 \alpha$ can activate HSCs by regulating autophagy. FIH1 has been shown to be involved in liver fibrosis by negatively regulating HIF-1 $\alpha$ expression [22]. In our study, bioinformatics analysis and luciferase reporter experiments indicated that FIH1 is a target of miR-125a-5p, which expands the repertoire of miR-125a-5p targets. Further studies revealed that miR-125a-5p promotes the activation and proliferation of HSCs by down-regulating FIH1 level. However, the functional role of miR-125a-5p in vivo requires further investigation.

Taken together, our findings indicate that miR-125a-5p can induce the activation and proliferation of HSCs, at least in part, by down-regulating FIH1. Possible use of this miRNA as a therapeutic target requires better knowledge of its effects on carcinogenesis.

\section{Acknowledgements}

The project received grants from the National Natural Science Foundation of China (Grant no. 81500458/H0317), Zhejiang Provincial Natural Science Foundation of China (Grant no.LY16H030012), Chinese Foundation for Hepatitis Prevention and Control-TianQing Liver Disease Research Fund Subject in 2014 (Grant no. TQGB20140064), and Second Scientific Project of Yinzhou District in 2014 (2014, No. 57).

\section{Disclosure Statement}

The authors have stated that there is no competing interest in this study.

\section{References}

1 Zhang X, Tan Z, Wang Y, Tang J, Jiang R, Hou J, Zhuo H, Wang X, Ji J, Qin X, Sun B: Ptpro-associated hepatic stellate cell activation plays a critical role in liver fibrosis. Cell Physiol Biochem 2015;35:885-898.

2 Xiao Y, Qu C, Ge W, Wang B, Wu J, Xu L, Chen Y: Depletion of thymosin beta4 promotes the proliferation, migration, and activation of human hepatic stellate cells. Cell Physiol Biochem 2014;34:356-367.

3 Graybill RM, Bailey RC: Emerging biosensing approaches for microRNA analysis. Anal Chem 2016;88:431450 .

4 Ono K: Functions of microRNA-33a/b and microRNA therapeutics. J Cardiol 2016;67:28-33.

5 Ge S, Xie J, Liu F, He J: MicroRNA-19b reduces hepatic stellate cell proliferation by targeting grb2 in hepatic fibrosis models in vivo and in vitro as part of the inhibitory effect of estradiol. J Cell Biochem 2015;116:2455-2464.

6 Yang JJ, Tao H, Hu W, Liu LP, Shi KH, Deng ZY, Li J: MicroRNA-200a controls nrf2 activation by target keap1 in hepatic stellate cell proliferation and fibrosis. Cell Signal 2014;26:2381-2389.

7 He Y, Huang C, Sun X, Long XR, Lv XW, Li J: MicroRNA-146a modulates tgf-beta1-induced hepatic stellate cell proliferation by targeting smad4. Cell Signal 2012;24:1923-1930.

8 Lakner AM, Steuerwald NM, Walling TL, Ghosh S, Li T, McKillop IH, Russo MW, Bonkovsky HL, Schrum LW: Inhibitory effects of microRNA 19b in hepatic stellate cell-mediated fibrogenesis. Hepatology 2012;56:300310.

9 Roderburg C, Urban GW, Bettermann K, Vucur M, Zimmermann H, Schmidt S, Janssen J, Koppe C, Knolle P, Castoldi M, Tacke F, Trautwein C, Luedde T: Micro-RNA profiling reveals a role for mir-29 in human and murine liver fibrosis. Hepatology 2011;53:209-218. 
10 Wang J, Chu ES, Chen HY, Man K, Go MY, Huang XR, Lan HY, Sung JJ, Yu J: MicroRNA-29b prevents liver fibrosis by attenuating hepatic stellate cell activation and inducing apoptosis through targeting pi3k/akt pathway. Oncotarget 2015;6:7325-7338.

11 Yu F, Lu Z, Huang K, Wang X, Xu Z, Chen B, Dong P, Zheng J: MicroRNA-17-5p-activated wnt/beta-catenin pathway contributes to the progression of liver fibrosis. Oncotarget 2016;7:81-93.

12 Odar K, Bostjancic E, Gale N, Glavac D, Zidar N: Differential expression of microRNAs mir-21, mir-31, mir203, mir-125a-5p and mir-125b and proteins pten and p63 in verrucous carcinoma of the head and neck. Histopathology 2012;61:257-265.

13 Nishida N, Mimori K, Fabbri M, Yokobori T, Sudo T, Tanaka F, Shibata K, Ishii H, Doki Y, Mori M: MicroRNA$125 a-5 p$ is an independent prognostic factor in gastric cancer and inhibits the proliferation of human gastric cancer cells in combination with trastuzumab. Clin Cancer Res 2011;17:2725-2733.

14 Hsieh TH, Hsu CY, Tsai CF, Long CY, Chai CY, Hou MF, Lee JN, Wu DC, Wang SC, Tsai EM: Mir-125a-5p is a prognostic biomarker that targets hdac4 to suppress breast tumorigenesis. Oncotarget 2015;6:494-509.

15 Gao W, Chan JY, Wong TS: Curcumin exerts inhibitory effects on undifferentiated nasopharyngeal carcinoma by inhibiting the expression of mir-125a-5p. Clin Sci (Lond) 2014;127:571-579.

16 Jiang L, Huang Q, Chang J, Wang E, Qiu X: MicroRNA hsa-mir-125a-5p induces apoptosis by activating p53 in lung cancer cells. Exp Lung Res 2011;37:387-398.

17 Jiang L, Huang Q, Zhang S, Zhang Q Chang J, Qiu X, Wang E: Hsa-mir-125a-3p and hsa-mir-125a-5p are downregulated in non-small cell lung cancer and have inverse effects on invasion and migration of lung cancer cells. BMC Cancer 2010;10:318.

18 Coppola N, Potenza N, Pisaturo M, Mosca N, Tonziello G, Signoriello G, Messina V, Sagnelli C, Russo A, Sagnelli E: Liver microRNA hsa-mir-125a-5p in hbv chronic infection: Correlation with hbv replication and disease progression. PLoS One 2013;8:e65336.

19 Zheng J, Zhou Z, Xu Z, Li G, Dong P, Chen Z, Lin D, Chen B, Yu F: Serum microRNA-125a-5p, a useful biomarker in liver diseases, correlates with disease progression. Mol Med Rep 2015;12:1584-1590.

20 Kaimori A, Potter J, Kaimori JY, Wang C, Mezey E, Koteish A: Transforming growth factor-beta1 induces an epithelial-to-mesenchymal transition state in mouse hepatocytes in vitro. J Biol Chem 2007;282:2208922101.

21 Yu F, Zheng J, Mao Y, Dong P, Lu Z, Li G, Guo C, Liu Z, Fan X: Long non-coding RNA growth arrest-specific transcript 5 (gas5) inhibits liver fibrogenesis through a mechanism of competing endogenous RNA. J Biol Chem 2015;290:28286-28298.

22 Hu J, Chen C, Liu Q, Liu B, Song C, Zhu S, Wu C, Liu S, Yu H, Yao D, Kang J, Zhu L: The role of mir-31/fih1 pathway in tgfbeta-induced liver fibrosis. Clin Sci (Lond) 2015;129:305-317.

23 Song C, Wu G, Xiang A, Zhang Q Li W, Yang G, Shi X, Sun S, Li X: Over-expression of mir-125a-5p inhibits proliferation in c2c12 myoblasts by targeting e2f3. Acta Biochim Biophys Sin (Shanghai) 2015;47:244-249.

24 Tong Z, Liu N, Lin L, Guo X, Yang D, Zhang Q: Mir-125a-5p inhibits cell proliferation and induces apoptosis in colon cancer via targeting bcl2, bcl2l12 and mcl1. Biomed Pharmacother 2015;75:129-136.

25 Banerjee S, Cui H, Xie N, Tan Z, Yang S, Icyuz M, Thannickal VJ, Abraham E, Liu G: Mir-125a-5p regulates differential activation of macrophages and inflammation. J Biol Chem 2013;288:35428-35436.

26 Li D, Kong C, Tsun A, Chen C, Song H, Shi G, Pan W, Dai D, Shen N, Li B: Mir-125a-5p decreases the sensitivity of treg cells toward il-6-mediated conversion by inhibiting il-6r and stat3 expression. Sci Rep 2015;5:14615.

27 Potenza N, Papa U, Mosca N, Zerbini F, Nobile V, Russo A: Human microRNA hsa-mir-125a-5p interferes with expression of hepatitis b virus surface antigen. Nucleic Acids Res 2011;39:5157-5163.

28 Copple BL, Bai S, Burgoon LD, Moon JO: Hypoxia-inducible factor-1alpha regulates the expression of genes in hypoxic hepatic stellate cells important for collagen deposition and angiogenesis. Liver Int 2011;31:230244.

29 Wang Y, Huang Y, Guan F, Xiao Y, Deng J, Chen H, Chen X, Li J, Huang H, Shi C: Hypoxia-inducible factor1alpha and mapk co-regulate activation of hepatic stellate cells upon hypoxia stimulation. PLoS One 2013;8:e74051.

30 Moon JO, Welch TP, Gonzalez FJ, Copple BL: Reduced liver fibrosis in hypoxia-inducible factor-1alphadeficient mice. Am J Physiol Gastrointest Liver Physiol 2009;296:G582-592.

31 Deng J, Huang Q Wang Y, Shen P, Guan F, Li J, Huang H, Shi C: Hypoxia-inducible factor-1alpha regulates autophagy to activate hepatic stellate cells. Biochem Biophys Res Commun 2014;454:328-334. 